\title{
Tracheobronchopathia osteochondroplastica: eine ungewöhnliche Ursache einer Retentionspneumonie
}

\begin{abstract}
Zusammenfassung: Ein 79 Jahre alter Patient ohne pulmonale Vorerkrankungen wurde mit den klinischen Zeichen einer Pneumonie und mit Hämoptysen aufgenommen. Das Fieber besserte sich trotz antibiotischer Therapie innerhalb der ersten sechs Tage nicht. In der Bronchoskopie, die daraufhin zum Ausschluss eines stenosierenden Prozesses durchgeführt wurde, zeigte sich eine Tracheobronchopathia osteochondroplastica mit dem charakteristischen makroskopischen Bild einer Tropfsteinhöhlentrachea. Direkt subglottisch fand sich ein subtotal stenosierender Befund. In den aus diesen Bereichen entnommenen Proben wurden zytologisch zunächst Zellen eines Adenokarzinoms nachgewiesen. Histologisch konnte jedoch die Diagnose der Tracheobronchopathia osteochondroplastica bestätigt werden, durch den Verlauf und begleitende radiologische Untersuchungen wurde ein Malignom ausgeschlossen. Der vorgestellte Fall zeigt, dass eine Tracheobronchopathia osteochondroplastica über lange Zeit und auch in fortgeschrittenen Fällen asymptomatisch verlaufen kann. Die Möglichkeit falsch positiver Zytologien ist differenzialdiagnostisch zu berücksichtigen. Die Kenntnis des typischen bronchoskopischen Befundes sowie die histologische Untersuchung sind von entscheidender Bedeutung, um die Diagnose verlässlich zu stellen.
\end{abstract}

Tracheobronchopathia Osteochondroplastica: An Uncommon Cause of Retention Pneumonia in an Elderly Patient: A 79 years old patient without preexisting pulmonary disease was admitted due to pneumonia and hemoptysis. Despite intravenous antibiotic therapy he did not recover and still suffered from fever and dyspnea six days later. Fiberoptic bronchoscopia was performed in order to exclude poststenotic pneumonia. However, macroscopically a „rock-garden“ trachea, the characteristic picture of osteochondroplastic tracheobronchopathy, was seen with multiple whitish irregularly shaped nodules in the trachea, except in the pars membranacea, involving both sides of the bronchial system and producing subtotal stenosis. Although cytologic examination suggested adenocarcinoma, histology confirmed the diagnosis of osteochondroplastic tracheobronchopathy. Repeated CT scans as well as control bronchoscopy served as a means of excluding simultaneous carcinoma. The case presented here demonstrates that even progressive tracheobronchopathy may remain asymptomatic for a long time until subtotal stenosis or impaired clearing mechanisms may lead to retention pneumonia. Cytologic exami-

Pneumologie 2001; 55: 563-567

(c) Georg Thieme Verlag Stuttgart · New York ISSN 0934-8387

\section{G.-C. Sutor, T. Glaab, C. Eschenbruch, H. Fabel}

Abteilung Pneumologie (Leiter Prof. Dr. med. H. Fabel), Zentrum Innere Medizin, Medizinische Hochschule Hannover nation may give false positive results suggesting malignant disease. However the typical macroscopic picture as well as histology should lead to the correct diagnosis.

\section{Einleitung}

Komplizierte Lobärpneumonien ohne Risikofaktor sollten Anlass zum Ausschluss eines malignen stenosierenden Prozesses geben. Ursache poststenotischer Pneumonien können jedoch auch benigne Grunderkrankungen und bestimmte Erreger sein [1]. So können atypische Mykobakterien oder Rhodokokken pseudotumoröse Läsionen induzieren, die den abhängigen Bronchus partiell oder komplett okkludieren und $\mathrm{zu}$ Infiltraten, z.T. mit Einschmelzungen führen [1-3]. Gerade im Fall mykobakterieller Infektionen existieren offenbar Übergänge und Verbindungen zu einer seltenen benignen Erkrankung, der Tracheobronchopathia osteochondroplastica $[2,4]$.

Bislang sind in der Literatur knapp 400 Patienten mit Tracheobronchopathia osteochondroplastica beschrieben [57]. Die Erkrankung tritt vergleichsweise selten auf und wird erst im späteren Lebensalter manifest, wenn bereits typische makroskopische Veränderungen aufgetreten sind. Im Mittel liegt das Alter der Patienten dann bei 63,5 Jahren. Viele Fälle werden erst post mortem diagnostiziert $[8,9]$. Die Inzidenz könnte daher höher liegen, als bislang angenommen, verläuft die Erkrankung doch sehr lange asymptomatisch [9].

Histopathologisch finden sich von den Knorpelspangen ausgehende knorpelige und knöcherne Proliferationen mesenchymalen Gewebes. Diese primär benignen Läsionen können aufgrund der zunehmenden Vulnerabilität der Schleimhaut und aufgrund einer Verlegung des freien Lumens zu Symptomen führen. Zwei Drittel der Patienten leiden an Husten, oft mit Hämoptysen, ungefähr die Hälfte an belastungsabhängiger Dyspnoe und ein Drittel an asthmatischen Beschwerden $[4,10]$. Während sich im konventionellen Röntgenbild des Thorax kaum typische Veränderungen finden lassen, kann die Diagnose bei einem Teil der Patienten mit Hilfe einer computertomographischen Untersuchung der Trachea gestellt werden $[8,9]$. Der diagnostische Standard und die Methode der Wahl zur Beurteilung von Ausmaß und möglichen Komplikationen ist jedoch die Bronchoskopie $[8,9]$.

In diesem Fallbericht eines 79-jährigen Patienten soll verdeutlicht werden, welche Ausmaße eine Tracheobronchopathie annehmen kann, bevor es zu einer klinisch manifesten Symp- 
tomatik kommt. Diese ist jedoch unspezifisch, und eine bronchoskopische und im Einzelfall auch die histologische Klärung sind für die Diagnosestellung und die differenzialdiagnostische Abgrenzung unerlässlich.

\section{Fallbeschreibung (P. S., 79 Jahre, männlich)}

\section{Anamnese}

Der Patient wurde mit den klinischen Zeichen einer Pneumonie und mit Hämoptysen stationär aufgenommen. Er berichtete über plötzlich aufgetretenes Fieber bis $40^{\circ} \mathrm{C}$, Schüttelfrost und retrosternale Schmerzen. Erst seit Beginn dieser Beschwerden kam es dann sekundär zu blutig tingiertem Auswurf. Vor zwei Jahren war er bereits aufgrund einer Pneumonie stationär behandelt worden, eine weitere Abklärung war damals nicht erfolgt. Pulmonale Vorerkrankungen waren nicht bekannt. Er war schon immer Nichtraucher, litt an einer langjährig bekannten koronaren Herzkrankheit, einer arteriellen Verschlusskrankheit und an einer Osteoporose.

\section{Status}

Der 79-jährige Patient war in deutlich reduziertem Allgemeinzustand, litt unter ausgeprägter Dys- und Orthopnoe und bot eine Lippen- und Akrozyanose. Der Ernährungszustand war gut, die Körpergröße lag bei $172 \mathrm{~cm}$, das Gewicht betrug $70 \mathrm{~kg}$ (BMI 23,7 kg/m²). Die Körpertemperatur lag bei $39,8^{\circ} \mathrm{C}$. Erstgradige Struma diffusa. Über den Lungen vesikulär-bronchiales Atemgeräusch mit grobblasigen Rasselgeräuschen über beiden Lungenmittel- und Unterfeldern, betont rechts dorso-lateral, mit beidseits nachweisbarer basaler Klopfschallabschwächung. Der Puls war tachykard, 108/min, arrhythmisch, und der nach Riva-Rocci gemessene Blutdruck betrug beidseits 200/100 mm Hg. Auskultatorisch fand sich ein 2/6 Systolikum mit p.m. über Erb. Die Untersuchung von Abdomen, Extremitäten und Gelenken war unauffällig, der Gefäßstatus zeigte abgeschwächte Fußpulse beidseits.

\section{Laborparameter}

Auffällig waren erhöhte Entzündungsparameter: CRP: 189 mg/l (Norm: < 7), BKS: 17/36 mm (Norm: 4/8), Leukozyten: $23700 / \mu l$ (88\% neutrophile Granulozyten). Erhöht waren ferner das MCV auf $97 \mathrm{fl}$ (Norm: 80-96) und das MCH auf 35 pg (Norm: 28-32) i.S. einer Makrozytose. Bei grenzwertig noch normalen Gammaglobulinen fand sich der Nachweis eines Paraproteins von $9 \mathrm{~g} / \mathrm{l}$. Erniedrigt waren: TSH: 0,15 mU/1 (Norm: 0,3-0,4) bei unauffälligen peripheren Schilddrüsenparametern, Gesamtprotein: $64 \mathrm{~g} / \mathrm{l}$ (Norm: $72-80$ ), Albumin: $34 \mathrm{~g} / \mathrm{l}$ (Norm: 38 -45).

Die arterielle BGA zeigte unter $31 \mathrm{O}_{2} /$ min eine respiratorische Partialinsuffizienz: $\mathrm{pO}_{2}$ 59,4 Torr, $\mathrm{pCO}_{2}$ 40,5 Torr, Sauerstoffsättigung: $91 \%$.

Bei einer Kontrolluntersuchung zehn Tage nach Beginn der Therapie hatte sich das TSH normalisiert, das CRP war auf $<7 \mathrm{mg}$ /l abgefallen, und auch die Leukozytose war nicht mehr nachweisbar (6800 Zellen/ $\mu \mathrm{l})$. Die arterielle Blutgasanalyse ergab jetzt einen $\mathrm{pO}_{2}$ von 99 Torr und einen $\mathrm{pCO}_{2}$ von 35,5 Torr (ohne $\mathrm{O}_{2}$-Gabe).
EKG

Das EKG zeigte einen Linkstyp bei einem Sinusrhythmus, Frequenz 70/min, einen AV-Block $\mathrm{I}^{\circ}$ sowie einen Linksschenkelblock ohne signifikante Erregungsrückbildungsstörungen.

\section{Spirometrie}

Die Vitalkapazität (VC) war leicht auf $2800 \mathrm{ml}$ (78\% der Norm) eingeschränkt, die $\mathrm{FEV}_{1}$ war mit $2200 \mathrm{ml}$ (79\% der VC) relativ zur VC normwertig. Die oszillatorisch gemessene Resistance lag mit 3,5 $\mathrm{cm} \mathrm{H}_{2} \mathrm{O} / \mathrm{l} / \mathrm{s}$ noch im Normbereich. Eine Bodyplethysmographie war aufgrund des schlechten Allgemeinzustandes nicht durchführbar. Nach zehn Tagen Therapie hatte sich die VC auf $3150 \mathrm{ml}$ normalisiert, die $\mathrm{FEV}_{1}$ lag mit $2400 \mathrm{ml}$ (76\% der VC) ebenfalls im Normbereich.

\section{Röntgen-Thorax}

Das Herz war nur mittelständig, noch normal groß, das Mediastinum nicht verzogen, Pleuraergüsse fanden sich nicht. Es fanden sich eine alveoläre Verschattung in Projektion auf den Mittellappen sowie feinfleckige disseminierte Verdichtungen in Projektion auf das apikale Unterlappensegment rechts bei ansonsten unauffälligem Lungenparenchym. Mediastinale oder intrapulmonale Raumforderungen ließen sich nicht abgrenzen.

\section{Bronchoskopie}

Bei insgesamt komplikationslosem Ablauf fanden sich nach Passage der Stimmritze über die gesamte Länge der Trachea linsen- bis kirschkerngroße exophytische Läsionen, die lediglich die Pars membranacea aussparten (Abb. 1a). Damit zeigte sich makroskopisch das charakteristische Bild einer Tropfsteinhöhlentrachea, pathognomonisch für eine Tracheobronchopathia osteochondroplastica. Die Veränderungen ragten z.T. deutlich in das freie Lumen hinein, und direkt subglottisch bewirkten sie eine Stenose mit einer ca. 30-40\%igen Trachealeinengung. Oberflächlich waren die Läsionen von weißlicher Farbe, teils auch gerötet und vulnerabel, und von harter Konsistenz. Die Schleimhautveränderungen setzten sich unverändert in beide Bronchialsysteme fort, mit Betonung der zentralen Anteile. Ab der Ebene der Segmentbronchi fand sich hingegen eine vollständig intakte Schleimhaut (Abb.1b). Insbesondere fand sich am Abgang des Mittellappens keine signifikante Stenose.

\section{CT-Thorax}

Die CT-Diagnostik, 7 Tage nach Diagnosestellung als Spiral-CT mit Kontrastmittelgabe durchgeführt, konnte die bekannte Mitellappenpneumonie in Rückbildung sowie feinfleckige Verdichtungen im rechten apikalen Unterlappensegment nachweisen. Eine malignitätsverdächtige Raumforderung fand sich nicht. Vor allem im Bereich der Trachealwand und der Wände des zentralen Bronchialsystems fanden sich noduläre bis flächige, in das Lumen hineinragende Kalzifikationen, die das Tracheallumen bereits sichtbar einengten (Abb. 2a und b). 

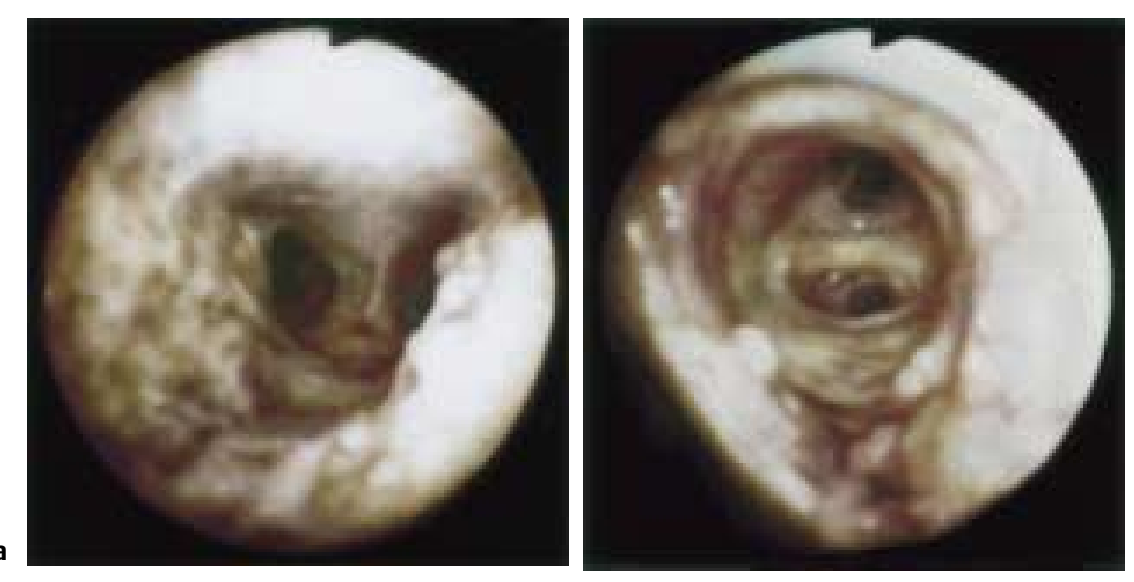

Abb. 1 Videobronchoskopie:

a Distale Trachea. Befund einer Tropfsteinhöhlentrachea mit endobronchialen Proliferationen mesenchymalen Gewebes.

b Peripheres Bronchialsystem. Subsegmentaufteilung der Lingula.
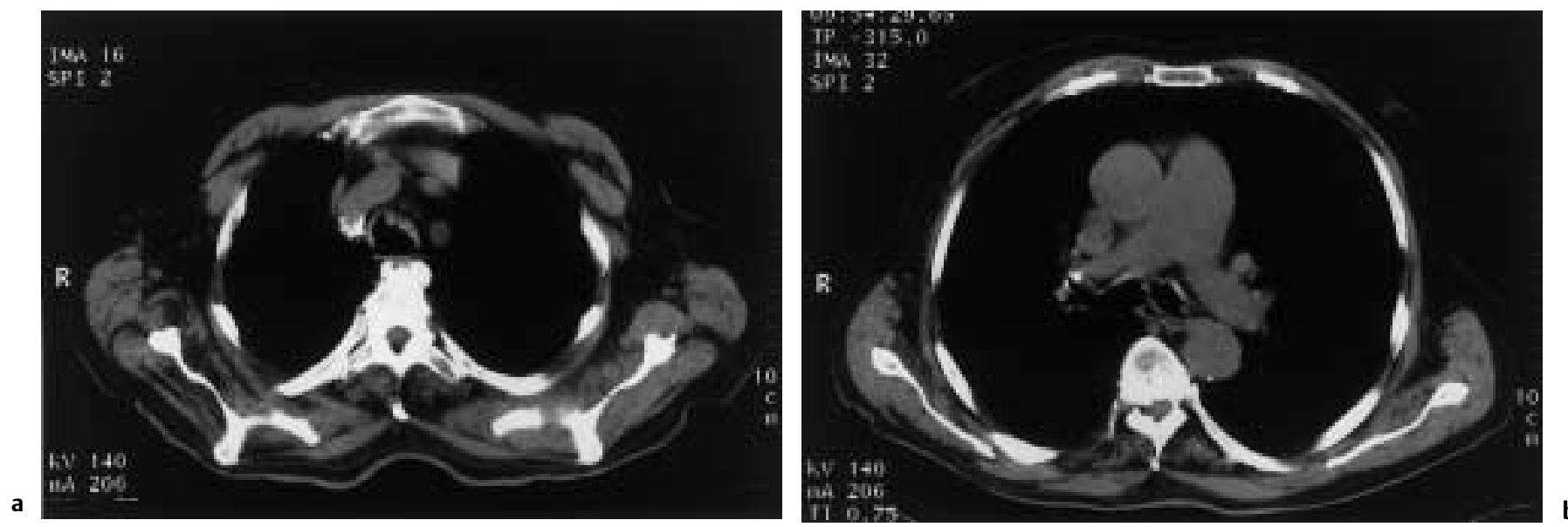

Abb. 2 CT des Thorax. Weichteilfenster mit Darstellung charakteristischer Kalzifikationen in der Trachea (a) und der zentralen Bronchien (b).

\section{Zytologie und Histologie}

Sowohl im Abstrichpräparat der Bronchusbiopsie als auch in der zytologischen Begutachtung eines Bürstenabstriches wurde primär die Diagnose eines gut differenzierten Adenokarzinoms, am ehesten eines Alveolarzellkarzinoms, gestellt. Dies aufgrund des Nachweises atypischer Zellen mit geringer Kernvolumenzunahme, Anisokaryozytose, exzentrischer Kernlagerung und der Bildung von azinären Gruppen. Zytochemisch war die alkalische Phosphatase-Reaktion stark positiv. Histologisch wurde bei Nachweis umschriebener Ossifikationen und Fibroseherde in der Bronchuswand die Diagnose einer Tracheobronchopathia osteochondroplastica gestellt.

\section{Therapie und Verlauf}

Das Fieber besserte sich unter antibiotischer Therapie mit Ceftriaxon, $1 \times 2 \mathrm{~g} / \mathrm{d}$, und Roxithromycin, $2 \times 150 \mathrm{mg} / \mathrm{d}$, erst nach Ablauf von sechs Tagen. Parallel bestand zu diesem Zeitpunkt jedoch noch eine unveränderte pulmonale Symptomatik mit ausgeprägter Ruhedyspnoe, Husten und Auswurf. Aufgrund dieses protrahierten Verlaufes und in Kenntnis der Tatsache, dass es sich um eine Rezidivpneumonie handelte, wurde das oben beschriebene CT des Thorax durchgeführt, und der Patient wurde bronchoskopiert. Der Patient wurde nach 10 Tagen mit einem Oralcephalosporin, Cefuroxim, $2 \times 500 \mathrm{mg} / \mathrm{d}$, in Kombination mit Roxithromycin für weitere 7 Tage behandelt. Hierunter kam es innerhalb von 21 Tagen zu einer vollständigen klinischen wie radiologischen Rückbildung der Pneumonie.

\section{Diskussion}

Der hier berichtete Fall einer Tracheobronchopathia osteochondroplastica verdeutlicht die typischen Charakteristika der Erkrankung. Makroskopisch konnten wir bei der Erstdiagnose ein weit fortgeschrittenes Bild diagnostizieren, das bereits zu einer subglottischen Trachealeinengung geführt hatte. Fast pathognomonisch ist, dass die Erkrankung über lange Zeit asymptomatisch blieb, und obwohl der Patient bereits zwei Jahre zuvor eine Pneumonie durchgemacht hatte, konnte die Diagnose erst sehr viel später bronchoskopisch gestellt werden. Nicht die Einengung des Tracheobronchialbaumes selbst, sondern vielmehr die gestörte mukoziliäre Clearancefunktion führte erst spät zu der Komplikation einer Retentionspneumonie. Dies ist, betrachtet man alle Fälle, über die bislang berichtet wurde, ein ausgesprochen typischer, gleichsam „benigner“ Verlauf der Erkrankung [4-10]. Inzwischen sind jedoch auch Patienten bekannt, bei denen ein rascher lokaler Progress zu einer klinisch signifikanten Stenose der Trachea oder zentraler Bronchien geführt hatte [11]. 
Gerade bei diesen Patienten sind natürlich vor allem Erkrankungen maligner oder infektiöser Genese auszuschließen, ansonsten umfasst die Differenzialdiagnose die Amyloidose, eine endobronchiale Sarkoidose, kalzifizierende Läsionen einer chronisch verlaufenden Tuberkulose, eine Papillomatose und eine tracheobronchiale Kalzinose.

Die Tracheobronchopathia osteochondroplastica ist hauptsächlich auf die zentralen Atemwege beschränkt, dennoch ist eine so ausgeprägte Beteiligung der oberen Trachea, auch wenn sie, wie bei unserem Patient, asymptomatisch blieb, sehr selten. Resultieren hieraus jedoch klinische Beschwerden i.S. einer extrathorakalen Stenose, kommen grundsätzlich eine Laser-, eine Argonbeamer oder eine Kryotherapie infrage [10]. Berücksichtigt werden muss bei der Entscheidung zu eingreifenderen Maßnahmen, dass die Erkrankung in der Regel benigne verläuft. So sind bislang noch keine Fälle beschrieben sind, in denen die Tracheobronchopathie direkte Todesursache gewesen wäre [10]. Die Prognose kann daher günstig gestellt werden, vorausgesetzt, die typischen Differenzialdiagnosen, insbesondere maligne Erkrankungen, konnten ausgeschlossen werden.

Während sich im konventionellen Röntgenbild des Thorax kaum typische Veränderungen finden lassen, kann die Diagnose bei einem Teil der Patienten mit Hilfe einer computertomographischen Untersuchung der Trachea gestellt werden. In unserem Fall konnten wir im konventionellen CT des Thorax bereits typische Läsionen finden, die unter Berücksichtigung des bronchoskopischen Befundes eindeutig zuzuordnen waren. Für eine sichere Diagnosestellung allein aus dem CT fehlen bislang jedoch kontrollierte und systematische Untersuchungen. In allen Einzelfallbeschreibungen wurde stets eine definitive bronchoskopische Klärung angeschlossen [15-19]. Der diagnostische Standard und die Methode der Wahl zur Beurteilung von Ausmaß und möglichen Komplikationen ist daher auch weiterhin die Bronchoskopie $[8,9]$.

Ergänzend zum makroskopischen Befund und v.a. dann, wenn die Veränderungen wenig ausgeprägt oder atypisch erscheinen, kann die Diagnose über die charakteristische Histologie gesichert werden. Hierbei ist es jedoch wichtig, die Verdachtsdiagnose zuvor zu stellen und dem Pathologen und dem Zytologen mitzuteilen. V.a. die Zytologie kann - wie bei unserem Patienten - die falsch positive Diagnose eines Adeno- oder Alveolarzellkarzinoms ergeben. Erst aufgrund der zusätzlichen Information über den makroskopischen Befund wurde die Diagnose i.S. einer Alveolarzellhyperplasie revidiert. Die Möglichkeit falsch positiver Zytologien ist daher in die Differentialdiagnose mit einzubeziehen, insbesondere dann, wenn nur eine Sputumzytologie vorliegt. $\mathrm{Zu}$ berücksichtigen ist jedoch, dass es durchaus eine Koinzidenz beider Erkrankungen gibt. Es sind bereits einige Patienten mit Adenocarcinomen und gleichzeitig bestehender Tracheobronchopathia osteochondroplastica beschrieben [12 - 14].

Histologisch finden sich typischerweise heterotope Ossifikationen in der Tracheal- bzw. Bronchialwand. Die Sensitivität liegt allerdings nur bei 60\% [10]. Aus diesem Grund kann die Histologie nur Zusatzinformation sein, die im Idealfall die Blickdiagnose sichert. In gleicher Weise kann die CT-morphologische Diagnostik in ausgeprägten Fällen helfen, die Diagnose zu bestätigen und über den Nachweis von Ossifikationen und Kalzifikationen eine Malignität der exophytischen Läsionen sehr unwahrscheinlich zu machen. Von einigen Autoren werden die Ossifikationen sogar als pathognomonisch für eine Tracheobronchopathia osteochondroplastica angesehen [15-19].

Typisch war bei unserem Patienten auch die unauffällige spirometrische Untersuchung. Die bislang hierzu publizierten Studien konnten trotz makroskopisch z.T. erheblicher Obstruktionen des zentralen Bronchialsystems keine spezifischen Veränderungen i.S. einer obstruktiven Ventilationsstörung dokumentieren [9-10]. Lediglich anhand der inspiratorischen Fluss-Volumen-Kurven bzw. durch die Bestimmung der FIV $_{1}$ kann z.T. eine variable extrathorakale Stenose diagnostiziert werden $[20,21]$.

Insgesamt verdeutlicht der hier vorgestellte Fall die Schwierigkeit der Diagnosestellung einer Tracheobronchopathia osteochondroplastica. Probleme können insbesondere die unspezifische Symptomatik sowie die Bewertung der Zytologie verursachen. Entscheidend bleibt der bronchoskopische Befund, wobei jedoch sowohl zytologische und histologische als auch mikrobiologische Differenzialdiagnosen berücksichtigt werden müssen. Die makroskopische Beurteilung unter der Bronchoskopie stellt weiterhin den Goldstandard der Diagnosestellung dar. Coputertomographie, Histologie und Zytolgie dienen der Bestätigung wie dem Ausschluss relevanter Differenzialdiagnosen.

\section{Literatur}

${ }^{1}$ Sutor GC, Fibrich C, Kirschner P, Kuske M, Schmidt RE, Schedel I, Deicher H. Endobronchial obstruction with poststenotic cavitating pneumonia due to Rhodococcus equi in HIV-infection. AIDS 1996; 10: 3-5

2 Baugnee PE, Delaunois LM. Mycobacterium avium-intracellulare associated with tracheobronchopathia osteochondroplastica. Eur Respir J 1995; 8: 180-182

${ }^{3}$ Sutor GC, Schuppert F, Freihorst J, Schätzle C, Schmidt RE. Primary combined immunodeficiency, growth retardation and disturbed sexual development. A novel syndrome of congenital impairment of the cellular immune system and the endocrine system. J Allergy Clin Immunol 1998; 102: 327-328

${ }^{4}$ Clee MD, Anderson JM, Johnston RN. Clinical aspects of tracheobronchopathia osteochondroplastica. Br J Dis Chest 1983; 77: $308-314$

${ }^{5}$ Mathlouthi A, Ben Rehouma C, Ben M'Rad S, Dumon JF, Labbene N, Driss B, Sellami M, Djenayah F. Tracheobronchopathia osteochondroplastica. Personal observation and review of the literature. Rev Pneumol Clin 1993; 49: 156- 162

${ }^{6}$ Meyer CN, Døssing M, Broholm H. Tracheobronchopathia osteochondroplastica. Respir Med 1997; 91: 499-502

${ }^{7}$ Saint-Blancard P, Natali F, Vaylet F, Countant G, L'Her P, Le Vagueresse R. Osteochondroplastic tracheobronchopathy. 5 cases. Rev Med Interne 1997; 18: 882 - 887

${ }^{8}$ Hodges MK, Isreal E. Tracheobronchopathia osteochondroplastica presenting as right middle lobe collapse. Diagnosis by bronchoscopy and computerized tomography. Chest 1988; 94: $842-844$

${ }^{9}$ Lundgren R, Stjernberg NL. Tracheobronchopathia osteochondroplastica. A clinical bronchoscopic and spirometric study. Chest 1981; 80: 706-709

${ }^{10}$ Nienhuis DM, Prakash UB, Edell ES. Tracheobronchopathia osteochondroplastica. Ann Otol Rhinol Laryngol 1990; 99: 689-694 
${ }^{11}$ Molloy AR, McMahon JN. Rapid progression of tracheal stenosis associated with tracheopathia osteo-chondroplastica. Intensive Care Med 1988; 15: 60-62

12 Kissler W, Wierich W, Meessen D. Tracheobronchopathia osteochondroplastica and carcinoma of the lung. Prax Klin Pneumol 1977; 31: 660-663

${ }^{13}$ Roggenbuck C, Hau T, de Wall N, Buss H. Simultaneous occurrence of tracheobronchopathia osteochondroplastica and mucoepidermoid carcinoma. Chirurg 1995; 66: 231 -234

${ }^{14}$ Fasske E. Tracheobronchopathia chondroosteoplastica with bronchial amyloidosis and bronchial cancer. Pathologe 1991; 12: $209-213$

${ }^{15}$ Matsuba T, Andoh K, Hirota N, Hara N. CT Diagnosis of Tracheobronchopathia osteochondroplastica. Respiration 2001; 68: 200

${ }^{16}$ Karlikaya C, Yuksel M, Kilicli S, Candan L. Tracheobronchopathia osteochondroplastica. Respirology 2000; 5: 377-380

${ }^{17}$ Manning JE, Goldin JG, Shpiner RB, Aberle DR. Case report: tracheobronchopathia osteochondroplastica. Clin Radiol 1998; 53: $302-309$

${ }^{18}$ Mariotta S, Pallone G, Pedicelli G, Bisetti A. Spiral CT and endoscopic findings in a case of tracheobronchopathiaochondroplastica. J Comput Assist Tomogr 1997; 21: 418-420

${ }^{19}$ Weber AL. Radiologic evaluation of the trachea. Chest Surg Clin N Am 1996; 6: 637-673

${ }^{20}$ Marlin GE. Tracheobronchopathia osteochondroplastica. Aust N Z J Med 1979; 9: 188-192

${ }^{21}$ Bergeron D, Cormier Y, Desmeules M. Tracheobronchopathia osteochondroplastica. Am Rev Respir Dis 1976; 114: 803 - 806
Dr. med. G.-C. Sutor

Zentrum Innere Medizin

Abteilung Pneumologie

Medizinische Hochschule Hannover

Carl-Neuberg-Straße 1

30625 Hannover 